\title{
Design and Preliminary Testing of the Volcanic Ash Plume Receiver Network
}

\author{
Nicholas Rainville, ScotT PALO, AND Kristine M. LARson \\ Department of Aerospace Engineering Sciences, University of Colorado Boulder, Boulder, Colorado \\ MARIO MATTIA \\ Istituto Nazionale di Geofisica e Vulcanologia, Osservatorio Etneo, Catania, Italy
}

(Manuscript received 5 October 2018, in final form 19 January 2019)

\begin{abstract}
The presence of volcanic ash in the signal path between a GPS satellite and a ground-based receiver strongly correlates with a decrease in GPS signal strength. This effect has been seen in data collected from GPS sites located near active volcanoes; however, the sparse placement of existing GPS sites limits the applicability of this technique as an ash plume detection method to relatively few well-instrumented volcanoes. This deficiency has motivated the development of a low-cost distributed sensor system based on navigation-grade GPS receivers, which can take advantage of attenuated GPS signals to increase the quality and availability of real-time ash plume observations during an eruption. This GPS-based system has been designed specifically to meet remote sensing needs while operating autonomously in difficult conditions and minimizing on-site infrastructure requirements. Prototypes of this system have undergone long-term testing and the data collected from this testing have been used to develop the additional processing steps necessary to account for the different behavior of navigation grade GPS equipment compared to the geodetic equipment used at existing GPS sites.
\end{abstract}

\section{Introduction}

When the Eyjafjallajökull volcano erupted in 2010, the resulting ash plume created far reaching hazards to aviation traffic. Fine ash from the eruption, which originated in Iceland, was transported by winds across northern Europe and as far south as Morocco (Petersen 2010). Due to the danger of ash ingestion by jet turbines, air traffic was either routed around these regions of dense airborne ash or it was shut down entirely. The ash density forecasts relied on for these decisions were provided by dispersion models run by Volcanic Ash Advisory Centers (VAACs). These forecasts can be improved and the impact of eruptions lessened by increasing the accuracy of the source terms used by the dispersion models (Mastin et al. 2017). Specifically, additional accuracy can be gained by providing better observations of parameters such as the eruption time, duration, plume altitude, mass eruption rate, and ash density (Witham et al. 2017).

Ash plumes can be directly detected by remote sensing instruments such as lidar, radar, and optical sensors,

Corresponding author: Nicholas Rainville, nicholas.rainville@ colorado.edu though these types of instruments are generally expensive and require additional infrastructure to operate. With the exception of radar, these instruments can also be affected by environmental conditions such as weather and time of day (Mackie et al. 2016). Radars operating at the appropriate frequencies are not affected by either, but due to the high cost for a radar system, weather radars tuned for precipitation are most frequently used for ash detection (Montopoli 2016). The VOLDORAD radar is an exception and is not hindered by precipitation, however, it is a nonscanning type radar with fixed pointing (Donnadieu et al. 2016). An alternative observation technique which maintains many of the same benefits of radar sensing is passive radio frequency $(\mathrm{RF})$ remote sensing. This technique relies on signals of opportunity from sources such as communications or navigation satellites in place of signals from a dedicated transmitter. Similar to radar, passive RF remote sensing instruments can operate in frequency bands which are not impacted by weather.

In some configurations, the global positioning system (GPS) can also serve as a passive remote sensing instrument for volcano monitoring. Initially geodesists used changes in the atmospheric delays on GPS carrier 
phase signals to infer the water content expected to be observed in a volcanic plume (Houlié et al. 2005). Later, Larson (2013) expanded the GPS plume sensing technique to instead use signal-to-noise ratio (SNR) GPS data, which were shown to be correlated with ash content rather than water content. In the next section we will summarize how GPS SNR signals have been used to infer volcanic ash plumes. We then describe a new implementation of the GPS plume sensing methodology, in which navigation-quality GPS systems are used instead of the higher-cost geodetic systems. Results from a long-term deployment of navigation-quality GPS are subsequently described.

\section{GPS ash plume detection}

GPS signals are broadcast in the L band, with GPS L1 and L2 signals transmitted at 1.5754 and $1.2276 \mathrm{GHz}$, respectively. At these frequencies, received signal power is not affected by atmospheric moisture or hydrometeors (Misra and Enge 2006). Importantly, GPS satellites also transmit at a stable and repeatable power level. The result is that changes observed in the received signal strength are dominated by the gain patterns of the transmitting and receiving antennas as well as the multipath reflection environment near the antenna. Since the ground tracks of GPS satellites repeat approximately every $12 \mathrm{~h}$ (Agnew and Larson 2007), the effect of both the antenna gain and persistent multipath interference can be filtered from the signal strength data through a comparison with a reference model based on the signal strength recorded on a different day. Any differences between the signal strength and the model can then be assumed to be caused by changes in the environment between the transmitting satellite and the receiving antenna. This signal strength can be determined from the carrier to noise ratio $\left(C / N_{0}\right)$ observables recorded by GPS receivers in order to monitor GPS signal reception. The $C / N_{0}$ data, which are given in units of $\mathrm{dB}-\mathrm{Hz}$, can be converted to the more commonly used SNR measure of signal strength in $\mathrm{dB}$ by subtracting a fixed offset based on the bandwidth of the receiver (Joseph 2010). Since the analysis performed in this paper is based entirely on differenced measurements of signal strength, the $C / N_{0}$ data will be used directly as a measure of relative SNR.

Shortly before a series of eruptions occurred at Redoubt Volcano in Alaska in 2009, four GPS sites were installed to monitor ground deformation. These sites provided the carrier phase observables used for positioning solutions, and these positions appeared to suddenly shift when an ash plume was present near the GPS site (Grapenthin et al. 2013). The sites also recorded $C / N_{0}$ measurements, which showed that the GPS signal strength decreased when a plume was present. This effect was developed into an ash detection algorithm by Larson (2013). The algorithm utilizes the $C / N_{0}$ data from a prior quiet day as a reference model and smooths it with a low-pass filter. If the $C / N_{0}$ during the event day differed by more than 2.5 times the standard deviation of the model and by an ad hoc minimum of $1.75 \mathrm{~dB}-\mathrm{Hz}$, an ash plume detection was flagged. Running this algorithm on the Redoubt Volcano data resulted in detections of the four largest explosive events during the active time period. This method was further refined in Larson et al. (2017) and resulted in detection of 13 out of 19 eruption events at Redoubt Volcano in 2009. The eruptions which failed to be detected either had small ash plumes or poor observability due to the GPS constellation geometry at the eruption time.

Similar techniques were used by Fournier and Jolly (2014) to detect an ash plume during a 2012 eruption at Tongariro TeMaari crater in New Zealand, by Aranzulla et al. (2014) for a 2013 eruption at Etna in Italy, and by Ohta and Iguchi (2015) for a 2012 eruption at the Sakurajima volcano in Japan. The results of these three studies further confirmed the relationship between the presence of ash in the GPS signal path and a decrease in signal strength. The observed drops in signal strength ranged from $2 \mathrm{~dB}-\mathrm{Hz}$ at Sakurajima to $10 \mathrm{~dB}-\mathrm{Hz}$ at Tongariro TeMaari.

Though the GPS network at Redoubt Volcano consisted of four GPS receivers, when a plume was detected it was generally seen only by one receiver and at most two satellites. At Redoubt Volcano this was due to the positioning of the receivers, which are located between 5 and $28 \mathrm{~km}$ from the vent, as well as the geometry of the constellation of GPS satellites. The GPS receivers at Tongariro TeMaari, Etna, and Sakurajima also encountered these limitations and for similar reasons. Improving GPS visibility into ash plumes, whether to detect smaller events or to gain additional observations of larger events, would require a denser network of receivers located closer to the erupting vent than is typical for existing GPS sites. This is a challenge when using the geodetic grade receivers necessary for ground deformation monitoring, both due to the high cost of the receivers and antennas as well as to the risk of placing them near an active volcanic vent.

\section{The VAPR network}

The need for additional distributed GPS signal strength measurements near active volcanic sites has driven the development of a dedicated plume sensing GPS system, the Volcanic Ash Plume Receiver (VAPR) system. This system is built around navigation grade GPS chips which 


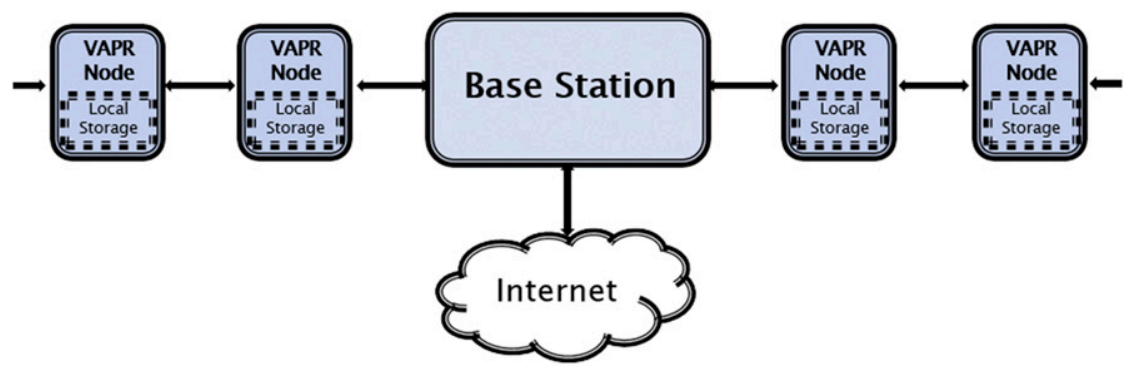

FIG. 1. The VAPR network. VAPR nodes connect to each other as well as to base stations through a flexible mesh network. The base stations collect the live SNR data from the nodes and send it though an Internet connection to an off-site server.

are paired with the power, communications, and data management subsystems necessary to operate as autonomous instruments. Additionally, the VAPR receivers are designed to operate as nodes in a mesh network to reduce the network infrastructure necessary at remote sites and to minimize losses due to the extreme conditions encountered during volcanic eruptions. This architecture removes the need for each receiver to have an external data connection and instead provides a dedicated wireless network to pass data between receivers. Base stations connected to the same network collect the SNR data and send it to an off-site Internet-connected server for processing as shown in Fig. 1.

\section{a. VAPR node design}

The VAPR network is composed of two parts, the receiver nodes and base stations. The receiver node includes the GPS receiver chip, a solar charging system, a radio, an SD card for storage, and a microcontroller for data management. The node circuit board in Fig. 2 illustrates these separate components. The receiver circuit board is housed in a weatherproof enclosure along with a battery, and is designed to be mounted to a mast along with a solar panel and antenna.

The VAPR node is designed around commercial GPS receiver chips. These chips are capable of reporting SNR data and cost less than $\$ 40$ per unit. Similar to the geodetic receivers, measuring signal strength is not their intended purpose. Unlike geodetic receivers, these chips are typically used for purposes such as automotive navigation, which do not require precise positioning, and they omit the capability to receive L2 frequency GPS signals. Additionally, at this price point, available receiver chips nearly universally support the NMEA 0183 standard. This standard specifies a message format which reports SNR data at a $1 \mathrm{~dB}-\mathrm{Hz}$ resolution. While observed decreases in signal strength caused by ash plumes have generally exceeded $1 \mathrm{~dB}-\mathrm{Hz}$, finer resolution is preferable as the detection technique is developed further.
This resolution limitation drove the selection of a chip that is a rare exception to the $1 \mathrm{~dB}-\mathrm{Hz}$ limit, the MediaTek MT3339, which supports a modified NMEA message reporting $0.1 \mathrm{~dB}-\mathrm{Hz}$ steps when used with a custom firmware. The downside to the MT3339 is that it only supports GPS and is not capable of receiving other global navigation satellite systems (GNSS) such as GLONASS or Galileo which could be used to provide additional signal strength measurements. For this reason, a second and optional receiver chip was also included in the design. The uBlox NEO-M8N was selected as a redundant receiver due to its support for multiple constellations, though it is limited in resolution to $1 \mathrm{~dB}-\mathrm{Hz}$. This secondary GPS receiver was not activated for initial testing.

Management of the data stream from the GPS chip is performed by an onboard microcontroller, which parses and reformats the SNR data into a radio packet for communication to a base station. Additionally, the microcontroller manages the local storage, power system, and housekeeping devices located on the node circuit board. The microcontroller is a 16-bit Microchip PIC and was selected due to the variety of input/output (I/O) buses required by the VAPR node as well as to minimize power consumption for operation with solar cells. The PIC is paired with a low-power radio for the communications link. The radio operates in either the $868-\mathrm{MHz}$ (Europe) or $900-\mathrm{MHz}$ (Americas) ISM bands and uses $182 \mathrm{~mW}$ to communicate up to $8 \mathrm{~km}$ line of sight. The trade-off for the low-power requirements is an extremely low data rate of $10 \mathrm{kbps}$. However, when reporting $1-\mathrm{Hz}$ data, only a 1-kbps connection is required an even this low bandwidth is sufficient.

The radio also includes built-in mesh networking support which allows the nodes to be networked in a daisy chain configuration to maximize the placement distance between network components (Digi 2006). An additional consideration was operation in different regulatory environments, since functionally similar radios are available in Europe, which operate in the license-free part of the European spectrum at $868 \mathrm{MHz}$. This radio 


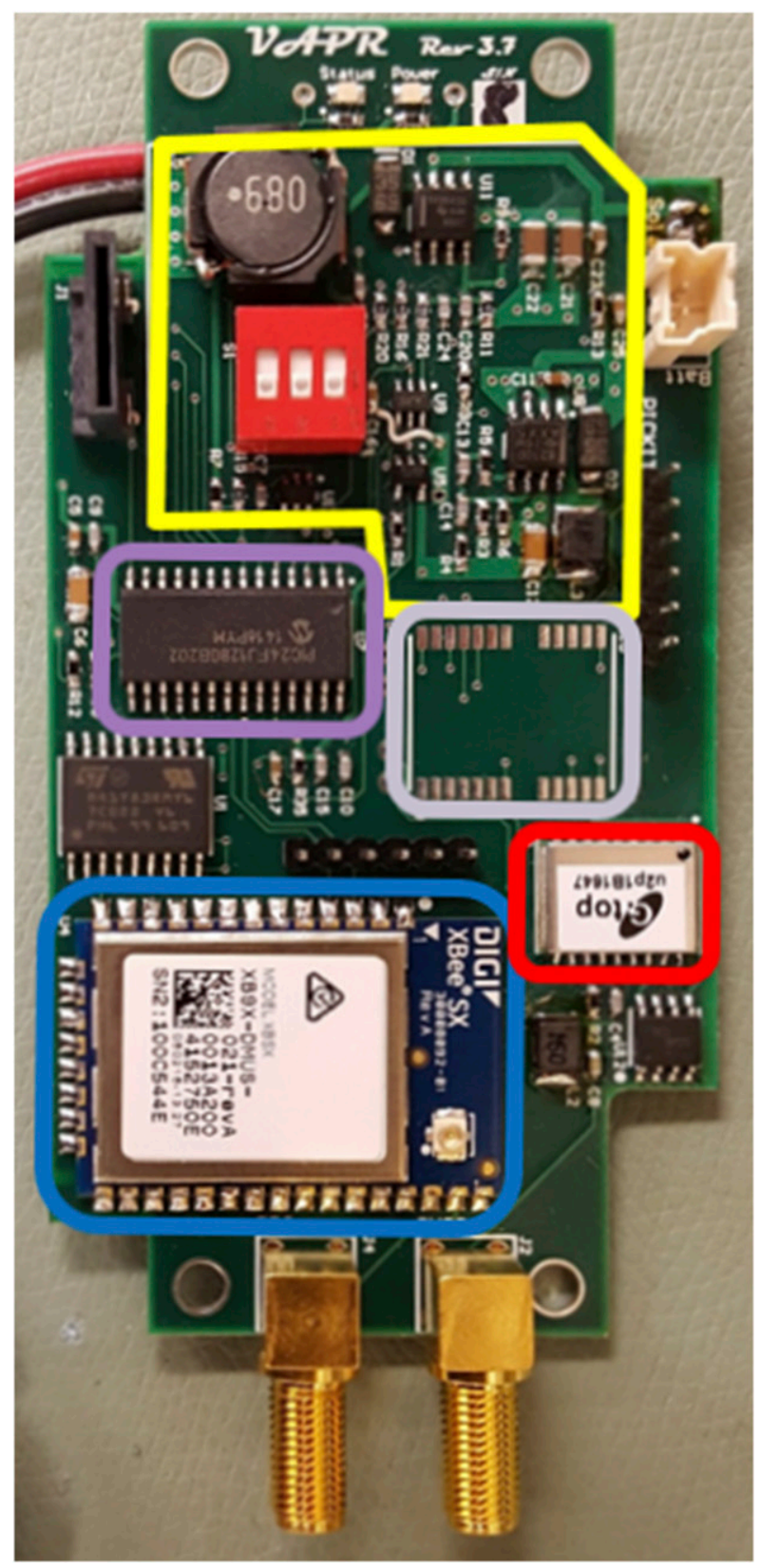

FIG. 2. The revision 3.7 VAPR node circuit board, which includes the power subsystem (yellow), the microcontroller (purple), the footprint for the uBlox GPS (silver), the GTop GPS (red), and the XBee radio (blue). The board measures $5 \mathrm{~cm} \times 10 \mathrm{~cm}$.

connection is required to monitor data from the node in real time, however onboard storage is also provided so data can be preserved in the case of network outages. An 8-GB micro SD card is used for this purpose due the limited physical space available on the node board. At a daily data rate of $4 \mathrm{MB}$, this provides over 5 years of storage.

In addition to data management, the microcontroller also controls the power regulation required to recharge the VAPR node. A 6-W solar panel is typically installed with the node and a maximum power point tracking (MPPT) algorithm links it to the built-in three-cell lithium-ion battery. Finally, the VAPR board also includes several housekeeping devices. The first is an external watchdog timer, which mitigates the effect of the software hanging and halting the microcontoller. The node also includes sensors to monitor the current board temperature as well as the battery voltage and charging state of the power system.

\section{b. VAPR antenna}

One of the most critical elements of the VAPR node is the GPS antenna. The SNR plume detection technique relies on finding SNR deviations from an expected model. This requires that the antenna provide a consistent and predictable gain pattern while rejecting undesired signals. High-quality antennas accomplish this by using large antenna elements and high-performance ground planes. Additionally, they are designed to receive only right-hand circular polarized signals and minimize the influence of left-hand polarized signals from specular reflections of the GPS signal. However, high-quality GPS antennas can cost thousands of dollars and would greatly exceed the cost of the rest of the node hardware. In contrast, small consumer grade antennas are readily available below $\$ 50$ and often include multiple constellation support. Though at this cost they provide poor multipath suppression, worse polarization performance, and asymmetrical gain patterns due both to the minimal ground element included with the antenna as well as the small size of the patch element relative to the GPS signal wavelength.

In an attempt to mitigate these performance issues, an inexpensive patch antenna was tested, the Taoglas MA111.c.lb.101, with an enlarged ground plane designed to improve low-elevation angle performance. The results from this configuration were mixed. While low-elevation angle signal suppression was improved, the increased size of the antenna reduced the reliability of the antenna in snow and high winds. Ultimately, reliability was prioritized over performance and the Trimble Bullet III antenna was selected for early testing of the VAPR network. Like the Taoglas, the Trimble uses a small patch as the radiating element, however the ground plane is fully self contained in a ruggedized radome cover which is steep enough to shed snow and present a minimal profile for wind loading. These antennas can be seen side by side in Fig. 3 .

\section{c. VAPR base station}

The second component of the VAPR network is the base station. The base station bridges the mesh network of VAPR nodes to an Internet connected server at the 


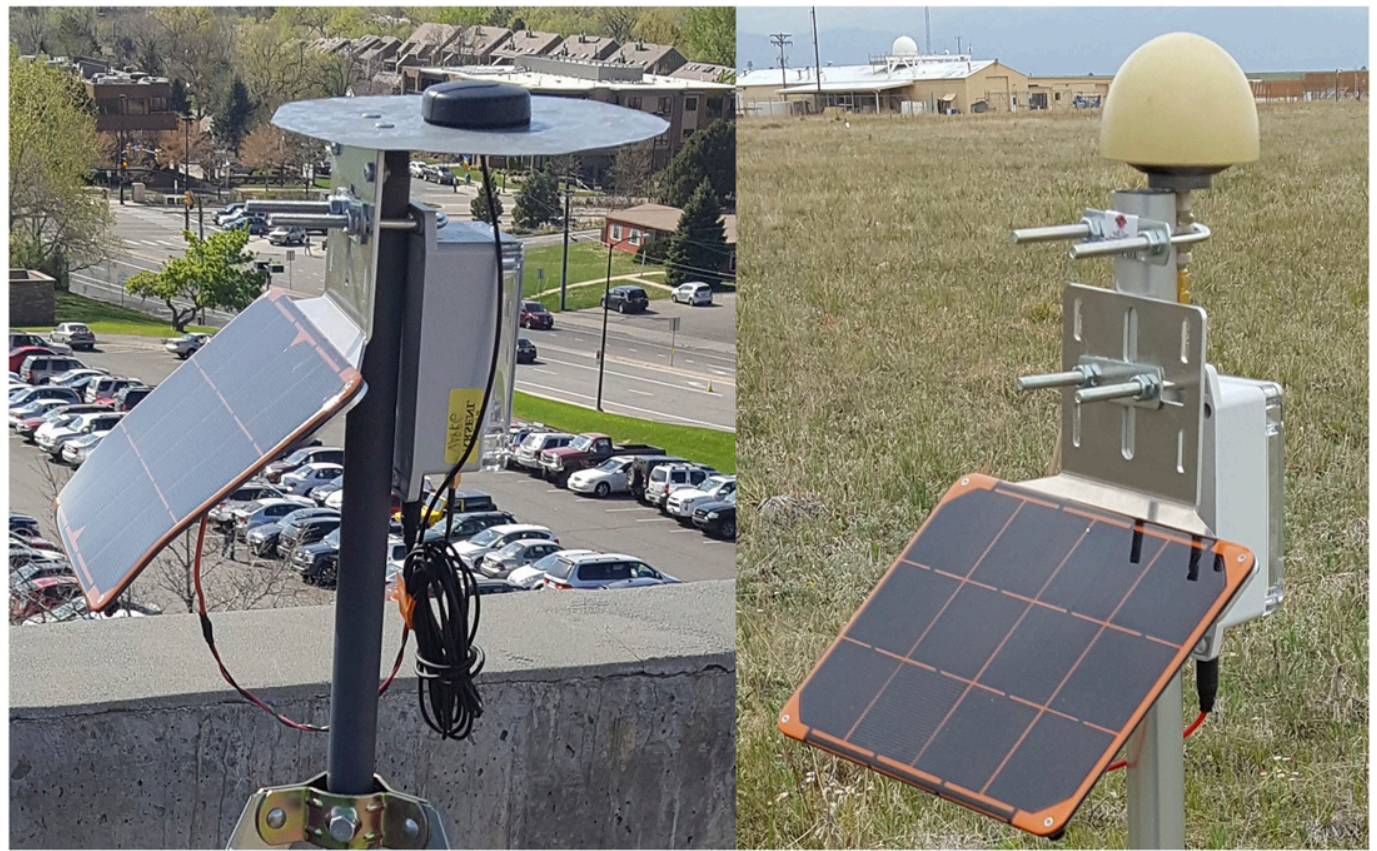

FIG. 3. (left) A Taoglass patch antenna with a 10-cm ground plane attached to a VAPR receiver in Boulder, Colorado. (right) A Trimble Bullet III antenna attached to a VAPR receiver at Marshall Mesa, Colorado.

University of Colorado Boulder (CU), which then processes and stores the SNR data. Each base station can communicate with four independent networks of VAPR nodes, so that multiple base stations can provide overlapping coverage of the node networks for redundant operation. Unlike the nodes the base station does require external infrastructure, specifically a network connection to the Internet. Initial testing sites were selected which had networks to provide the bandwidth for the combined 1-kbps per node data stream. This was due to the desire to capture $1-\mathrm{Hz}$ SNR data for remote processing. However, the infrastructure requirements could lessen as the detection algorithm is developed further. The base station has the processing capacity

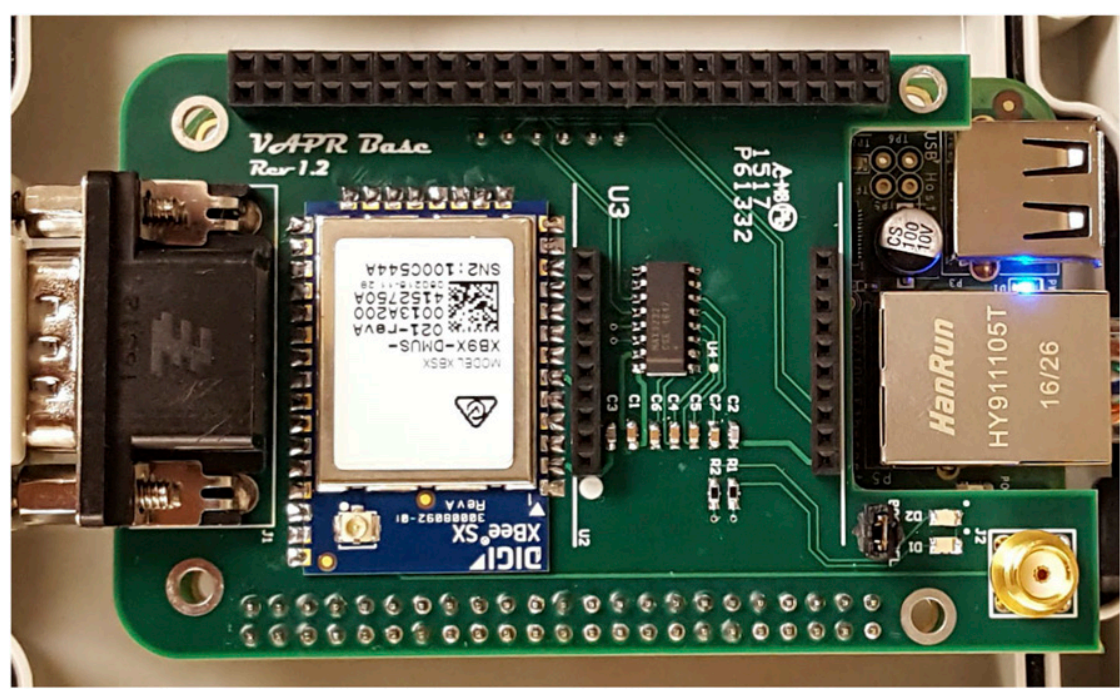

FIG. 4. The VAPR base station daughter-board, revision 1.2. The primary XBee radio is installed in the left footprint with a socket available for a second radio immediately to the right. The board measures $9 \mathrm{~cm}$ by $5 \mathrm{~cm}$. 
TABLE 1. GPS receivers used for SNR remote sensing comparison.

\begin{tabular}{|c|c|c|c|c|c|c|c|c|}
\hline Station & Description & Receiver & Antenna & Frequency & Location & Latitude & Longitude & Altitude \\
\hline $\mathrm{RX}$ & $\mathrm{VA}$ & Т3339 & III & & & 39 & 105 & \\
\hline MFLE & Geodetic & Trimble NetRS & Trimble Choke Ring & $\mathrm{L} 2 \mathrm{C}$ & Marshall & $39.9476^{\circ} \mathrm{N}$ & $105.1941^{\circ} \mathrm{W}$ & 1729.4 \\
\hline
\end{tabular}

necessary to calculate detections from the node data on board and reduce the network bandwidth requirement, which could allow for installations with low data rate cellular or satellite network connections. Additionally, since the base station components were selected with low power requirements in mind, the external power currently supplied by the testing sites could later be replaced by solar power.

The VAPR base station is designed around a BeagleBone Black (BBB) single board computer. This board is based on a 1-GHz 32-bit ARM processor and provides a significant increase in processing performance compared to the 8-MHz 16-bit PIC used for the VAPR nodes. The BBB runs a full Debian Linux distribution, which provides a complete transmission control protocol/Internet protocol (TCP/IP) stack as well as networking utilities for connecting to the $\mathrm{CU}$ server. While the base station consumes more power than the VAPR nodes, at $2.5 \mathrm{~W}$ it uses far less than typical desktop PCs, which operate at hundreds of watts. Since the BBB board design is open source, multiple versions from multiple manufacturers are available and the exact model used by the VAPR network can be selected based on the particular network and environmental requirements at the field site. While this satisfies the need for a networking device to connect to the Internet, the communication radios which connect to the nodes must be added on a custom daughterboard as seen in Fig. 4. This board contains a surface mount XBee radio, headers for a second through-hole mount radio, an RS-232 serial port for debugging, and status indicators. If additional radios are required, a second daughter board can be connected to the pass-through header on the top of the board to bring the total number of radios per base station to four.

\section{Testing results}

Initial testing of the VAPR system focused on verifying the reliability of a single VAPR node as well as confirming the suitability of the receiver and antenna combination for SNR remote sensing. The single node campaign led to design revisions which improved reliability of the nodes, particularly by improving the power system performance. This supported a shift into full network testing which included a base station and multiple nodes communicating in real time at a Colorado test site. The single node testing as well as the network testing was performed at Marshall Mesa in Boulder County, Colorado. This site is located on a 77-acre field used for instrumentation testing and operation. An operational geodetic GPS site is installed at the site and is managed by UNAVCO with a publicly available dataset (Braun 2011).

As part of the network testing, six receivers were cycled through the experimental configuration at Marshall Mesa. Operating this network uncovered the performance limits of the internode communications radios, which were designed for long-distance operation but required directional antennas to meet the advertised link distances. Omnidirectional wire antennas, while simple and robust, could only be used for subkilometer links while longer node-to-node links required more complex directional Yagi antennas.

These lessons were incorporated into the VAPR node design until reliable operation of the network was achieved. The following examples will focus on the VAPR node RX11, which includes these improvements. A full description of the receivers used for the following comparisons are listed in Table 1. Note that while the VAPR Nodes receive only L1 coarse/ acquisition (C/A) GPS signals, the geodetic receiver

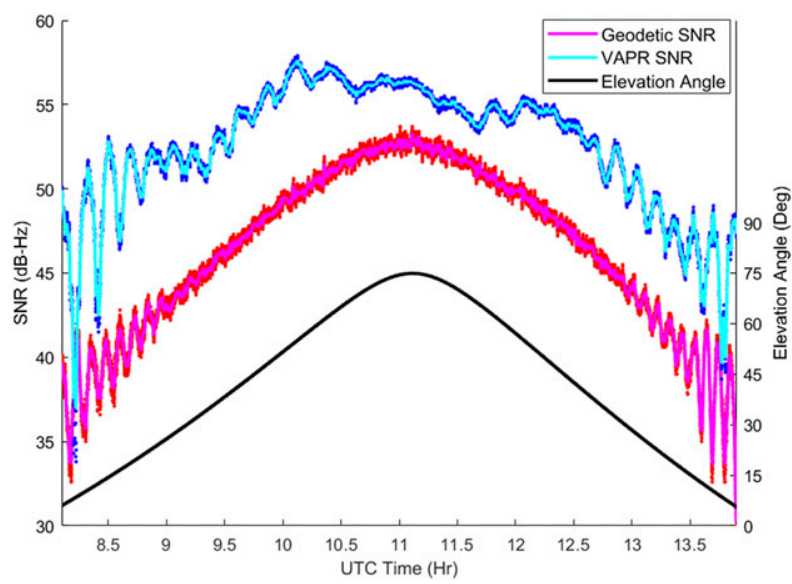

FIG. 5. SNR from PRN12 on day 345 (2017) showing the elevation dependence of the GPS antenna gain pattern. The L2C SNR from the geodetic receiver is shown in red with the $\mathrm{L} 1 \mathrm{C} / \mathrm{A}$ SNR from the VAPR receiver in blue. Five-minute moving averages are shown in cyan and magenta. The elevation angle of the GPS satellite is in black. 


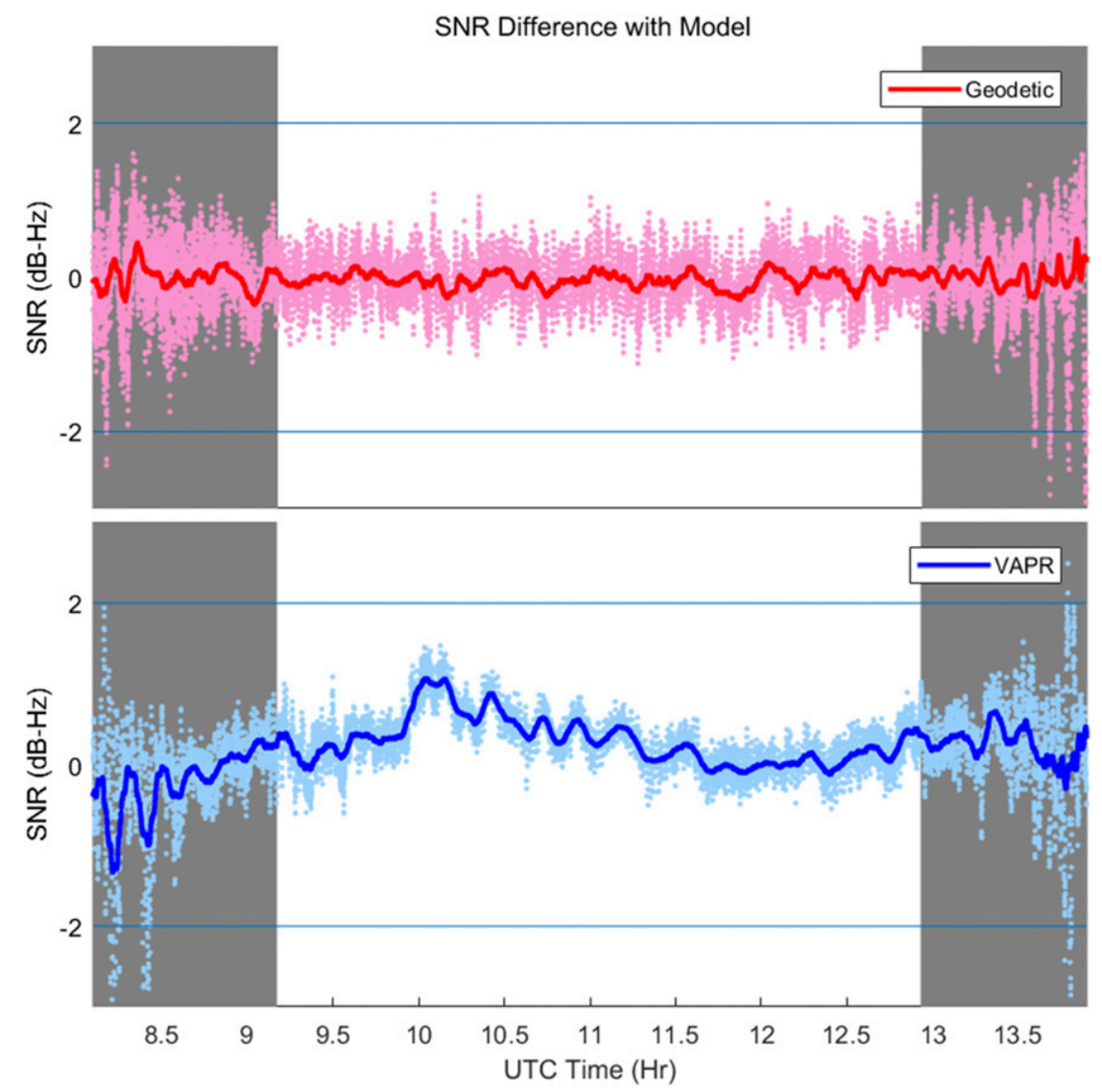

FIG. 6. The level 1 SNR difference from PRN12 on day 345 (2017) based on the algorithm described in section 2 . The lighter points show the $1-\mathrm{Hz}$ difference while the darker lines represent a 5-min moving average. Regions shown in gray occurred when the elevation angle of the GPS satellite was between $5^{\circ}$ and $30^{\circ}$.

records L1 C/A, L2P, and L2C SNR data. Since the L1 $\mathrm{C} / \mathrm{A}$ results are known to be problematic for the Trimble NetRS receiver (Larson and Nievinski 2013), L2C data have been used for comparisons instead.

\section{a. Comparison with geodetic GPS receivers}

While the consumer navigation receiver and small patch antenna used with the VAPR node is not expected to replicate the performance of larger and more expensive GPS receivers, comparing SNR data from the node against SNR data from geodetic sites provides an understanding of whether the performance would be sufficient for volcanic ash plume sensing. Based on the results from Redoubt Volcano, Tongariro TeMaari, Etna, and Sakurajima, a $2 \mathrm{~dB}-\mathrm{Hz}$ detection threshold was selected for this evaluation. Since the test site is not located near volcanic activity, exceeding this threshold is equivalent to a false detection.

A comparison made between the VAPR node and the geodetic receiver can be seen in Fig. 5. The data were collected on 11 December 2017 when the ground surface was dry and clear. While the VAPR SNR is generally higher than the geodetic SNR, these results illustrate the difference in gain pattern quality between the two antennas. The behavior of the L2C SNR data collected from the geodetic receiver is generally smooth and shows rapid $C / N_{0}$ variations only when the satellite is less than $30^{\circ}$ above the horizon, beyond that the SNR increases gradually as the elevation angle increases. In contrast, the L1 C/A SNR on the VAPR receiver shows the rapid SNR variations due to multipath continuing to higherelevation angles. Additionally, there is an asymmetrical pattern to the SNR data due to the gain pattern of the small patch antenna.

This structure is not necessarily a problem and as long as the SNR from the navigation grade receiver repeats day to day it can still be used as a reference for finding SNR variations caused by ash attenuation. Based on that assumption, a model of the expected SNR can be made from the observations recorded on a quiet day. The SNR 
model used in the following analysis is based on the previous day's observed SNR from each satellite. The SNR data are time shifted to account for the ground track repeat time and smoothed by a 10 -s moving average to remove high-frequency noise. This model represents the expected SNR for the site including local multipath interference as well as the unique gain effects of the particular antenna paired with the receiver. The model is then subtracted from the current SNR, which results in a difference referred to as the level 1 difference.

Figure 6 shows the level 1 difference for both the geodetic site and the VAPR receiver. The difference calculated using the VAPR receiver SNR shows more variation than the geodetic difference, but during this time period it stays well within the $2 \mathrm{~dB}-\mathrm{Hz}$ ash detection threshold. However, this time period is a best case sample of the SNR data from the VAPR receiver. While this level of data processing could be adequate to detect plumes with the geodetic receivers, applying it to the VAPR data over the course of a week results in Fig. 7, which shows the maximum difference per minute taken from all satellites above $30^{\circ}$ in elevation angle, again using the preceding day as a reference model. In this case the level 1 difference regularly exceeds $2 \mathrm{~dB}-\mathrm{Hz}$ and decreases in SNR caused by volcanic ash would be difficult to detect.

\section{b. SNR difference processing}

While the Mediatek MT3339 receiver used in each VAPR node reports SNR measurements which appear to compare well across short time periods, over longer periods the SNR can drift by several dB-Hz. This is especially problematic when the receiver periodically resets, an action taken in early versions of the firmware to prevent unexpected system hangs from limiting data collection and which can be seen in Fig. 8. The SNR has drifted and is biased lower than the prior days SNR, until at hour 2145 when the receiver performs a scheduled reset. After this reset the SNR returns to the same bias as the day before. While in this example the reset resolves the drift in SNR between the two days, it is just as likely to exacerbate the difference. This creates problems for any ash detection algorithm which is comparing signal strength across multiple days, such as the level 1 difference.

Fortunately, we have observed that the drifting bias in the recorded SNR measurement is consistent across all satellites and can be removed by adding an additional processing step, resulting in the level 2 difference. This algorithm follows the steps listed below:

1) Subtract the prior day model from the SNR, resulting in the level 1 difference.

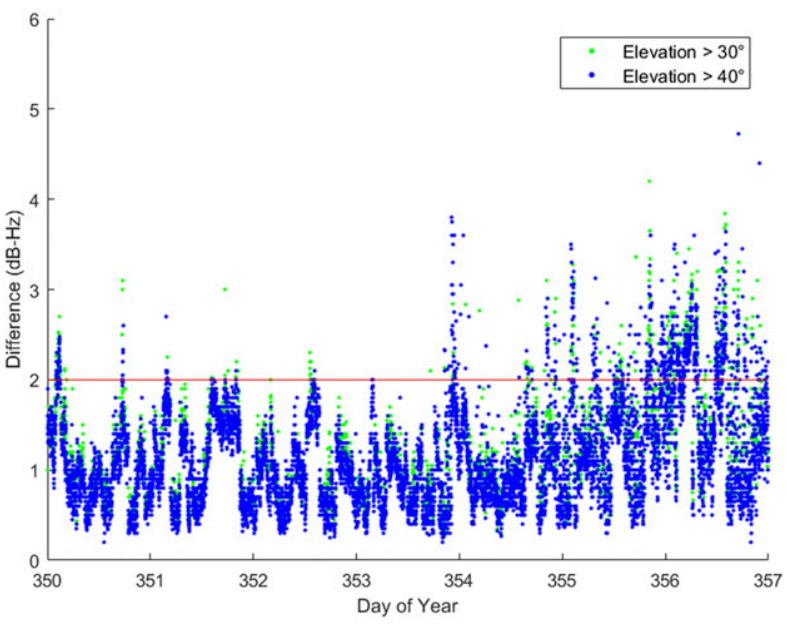

FIG. 7. The maximum difference per minute based on the level 1 algorithm for the VAPR receiver over days 350-357 (2017) for all satellites. Green markers represent a difference taken when a satellite was between $30^{\circ}$ and $40^{\circ}$ in elevation, blue when the satellite was above $40^{\circ}$. The majority of large differences occur at higher-elevation angles, indicating they are not entirely due to multipath interference.

2) Calculate the bias adjustment value by taking the median of the level 1 difference at each epoch across all high-elevation satellites.

3) Add the bias adjustment to the level 1 difference for each satellite, resulting in the level 2 difference.

The result of this processing is shown in Fig. 9. This plot covers same time period as Fig. 8 and the change in SNR bias when the receiver resets at hour 2145 is apparent when the lighter colored level 1 difference jumps back to near zero at hour 2145. The darker colored level 2 results are more consistently near zero due to the additional processing step, though the noise unique to each channel is still visible. The improved long-term performance when using level 2 differences can be seen in Fig. 10, where the maximum difference per minute rarely exceeds $2 \mathrm{~dB}-\mathrm{Hz}$ except on days 354, 355, and 356 . Overall, this decreases the percentage of minutes above the threshold from $8.68 \%$ to $0.14 \%$ of the total.

\section{c. Multipath interference mitigation}

The increased noise in the SNR difference on days 354-356 is due to multipath interference, which can occur when a reflected GPS signal combines with the GPS signal traveling directly from the satellite. The additional distance traveled by the reflected signal causes it to be delayed in phase compared to the direct signal, and it can then either constructively increase or destructively decrease the overall signal strength measured at the receiver. If either the position or the reflectivity of the reflecting surface changes, this interference will 


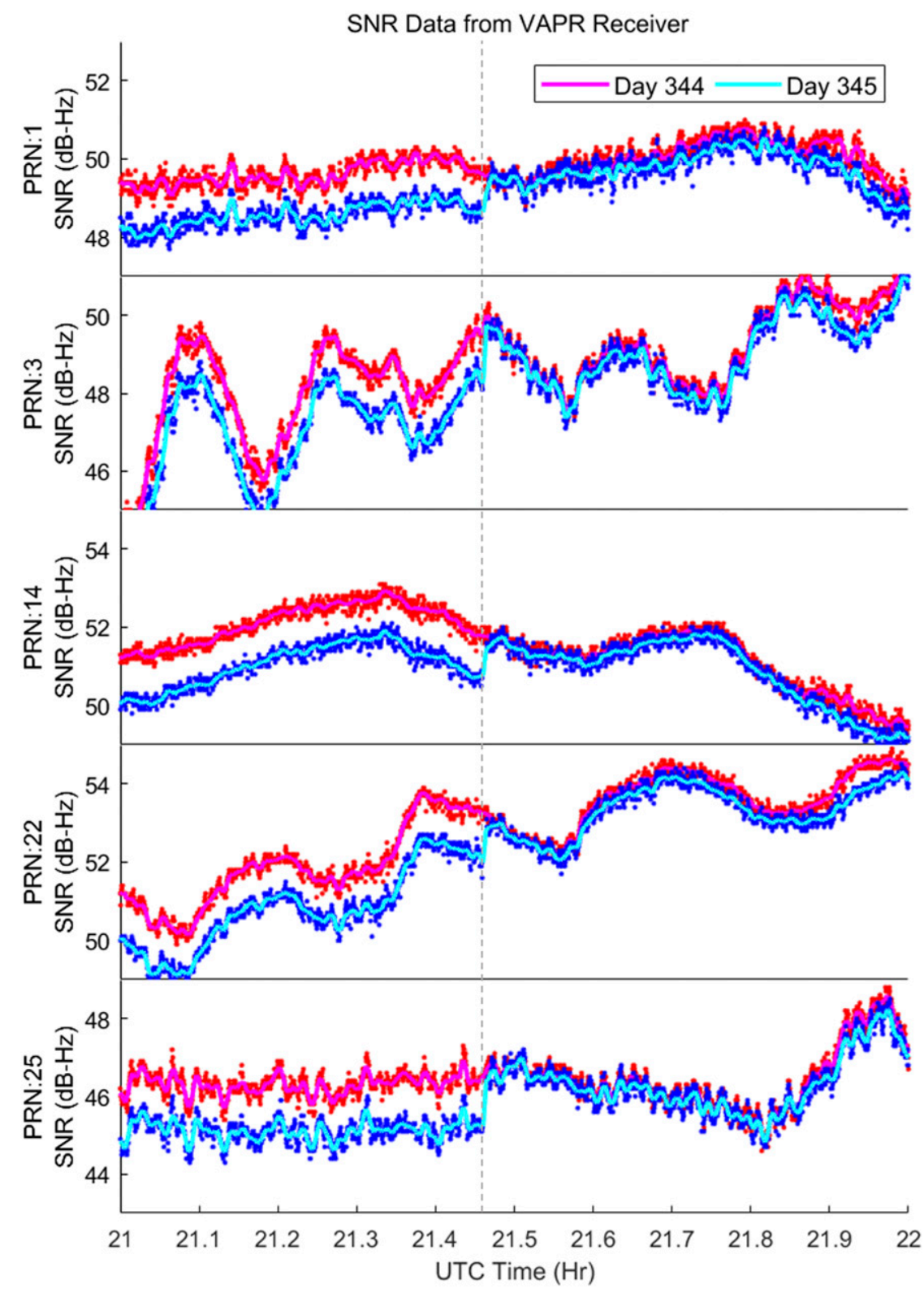

FIG. 8. SNR data from the VAPR receiver on days 344 and 345 (2017). All satellites above $30^{\circ}$ are shown, with the data from day 344 in red and day 345 in blue. A 5-min moving average of the SNR is shown in magenta and cyan. Between hour 21.4 and 21.5 a rapid jump in SNR occurs on all satellites, marked by the dotted line.

change as well and will show up in the SNR difference. When multipath reflection sources are azimuth dependent, such as structures or vehicles, the change in interference can be mitigated in software by applying a third level of processing which masks the SNR data by azimuth. However, this is only practical when the offending region is narrow and the masked azimuths are unlikely to observe ash. Often the interference will be in the direction of the vent, or in the case of ground reflections, from all azimuths and masking will not be beneficial.

One common reason for multipath interference to change between consecutive days is when the ground reflectivity and height changes due to snowfall. The impact of the ground reflection on the observed SNR can be seen even in the geodetic data as an elevation angle dependent sinusoid present at low satellite elevations. Snow reflects RF signals at GPS frequencies 


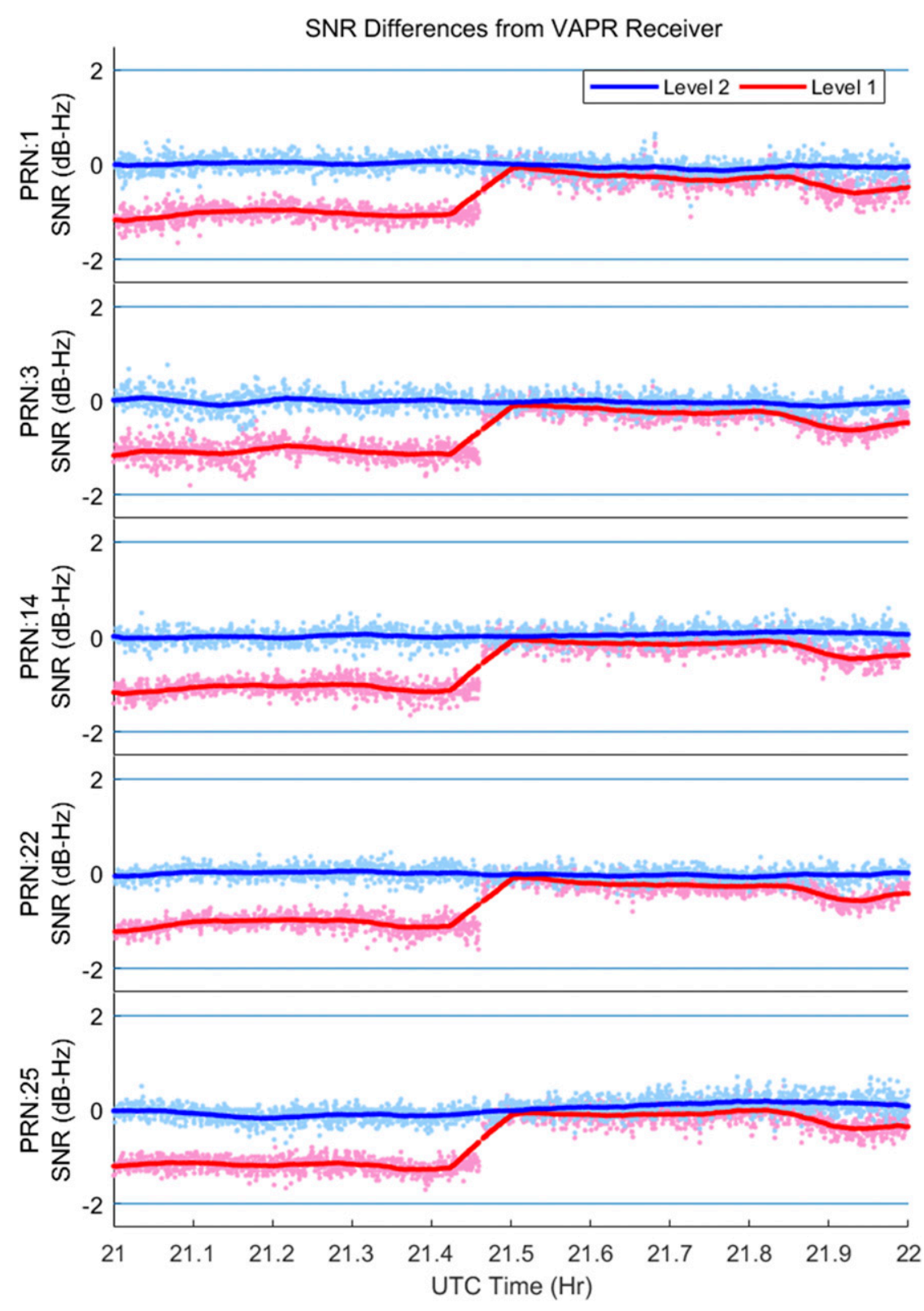

FIG. 9. The difference between the VAPR SNR on day 345 (2017) and a prior day model based on day 344 . All satellites above $30^{\circ}$ elevation are shown, with the level 1 difference in red and the level 2 difference in blue. The lighter markers show the 1-Hz difference, the darker line has been smoothed with a 5-min moving average. The rapid jump in SNR seen in Fig. 9 disappears when using the level 2 difference algorithm.

differently than bare soil and can change the amplitude of the ground reflection interference seen at the receiver. As snow depth increases, the relative height to the antenna decreases and the frequency of the interference sinusoid changes as well. While this effect can be desirable for snow depth sensing (Nievinski and Larson 2014a), it can also hide decreases in SNR due to ash attenuation.
With geodetic quality antennas the interferometric effect of ground reflections is generally only seen on signals arriving from satellites within $30^{\circ}$ of the horizon. For receivers close to an eruption, SNR data from higher-elevation angles are of the most interest due to the height of the ash plume relative to the receiver, so the impact of this effect is minimized. In contrast, the small patch antenna used by the VAPR system is less 


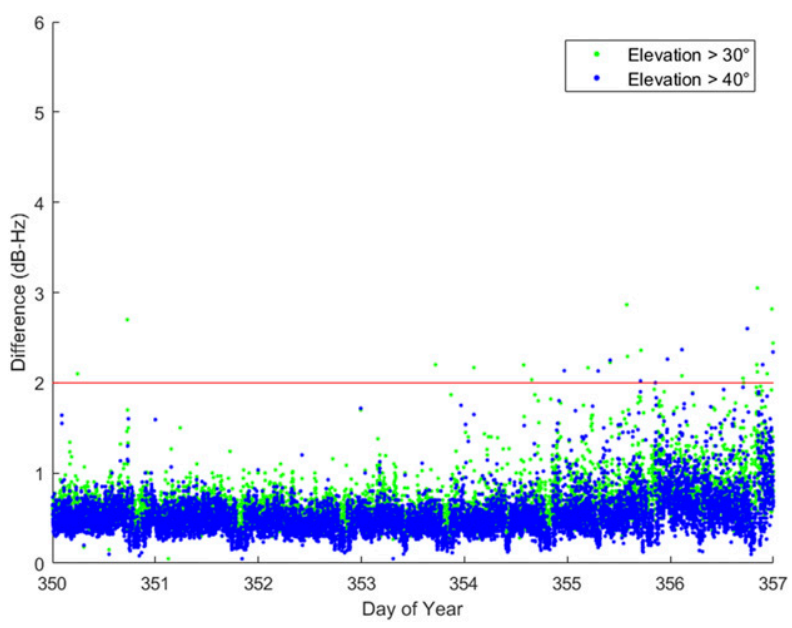

FIG. 10. The maximum difference per minute using the level 2 algorithm for the VAPR receiver on days 350 through 357 (2017) for all satellites. Green markers represent a difference taken when a satellite was between $30^{\circ}$ and $40^{\circ}$ in elevation, blue when the satellite was above $40^{\circ}$. The magnitude of the differences has decreased compared to Fig. 7 with larger differences occurring more commonly on satellites at low-elevation angles.

robust and the interference is still seen at higher-elevation angles. This is visible in the differenced SNR data in Figs. 11 and 12 which show the level 2 SNR differences on a dry day as well as a day which received snowfall.
To verify that the increased variability of the SNR to model difference was caused by snow, the SNR was further processed to calculate the ground reflection height based on the technique used by Nievinski and Larson (2014b). The left panel of Figs. 13 and 14 plot the SNR as a function of the elevation angle after removal of a fourth-order polynomial to account for the change in antenna gain pattern due to satellite elevation. With the geodetic data, the interference oscillations disappear above $30^{\circ}$, while for the VAPR data they remain strong at higher-elevation angles. The right panel of Figs. 13 and 14 show the same data converted into a power spectrum and referenced to height. Clear peaks can be seen on the dry day, which represent the height of the antenna above the bare ground surface. On the day with snow the height decreases on both receivers by roughly $15 \mathrm{~cm}$, which matches the snow depth record for that day (National Oceanic and Atmospheric Administration 2018).

The immediate consequence of this variability is that care needs to be taken when detecting ash plumes during periods of active snowfall or a melting snowpack. A change in the interference pattern due to new or different reflections could either trigger a false detection or obscure a real plume. For initial development and system testing these times can be excluded, however resolving this error source by improving the antenna performance is a necessity for fielding an operational plume detection system.

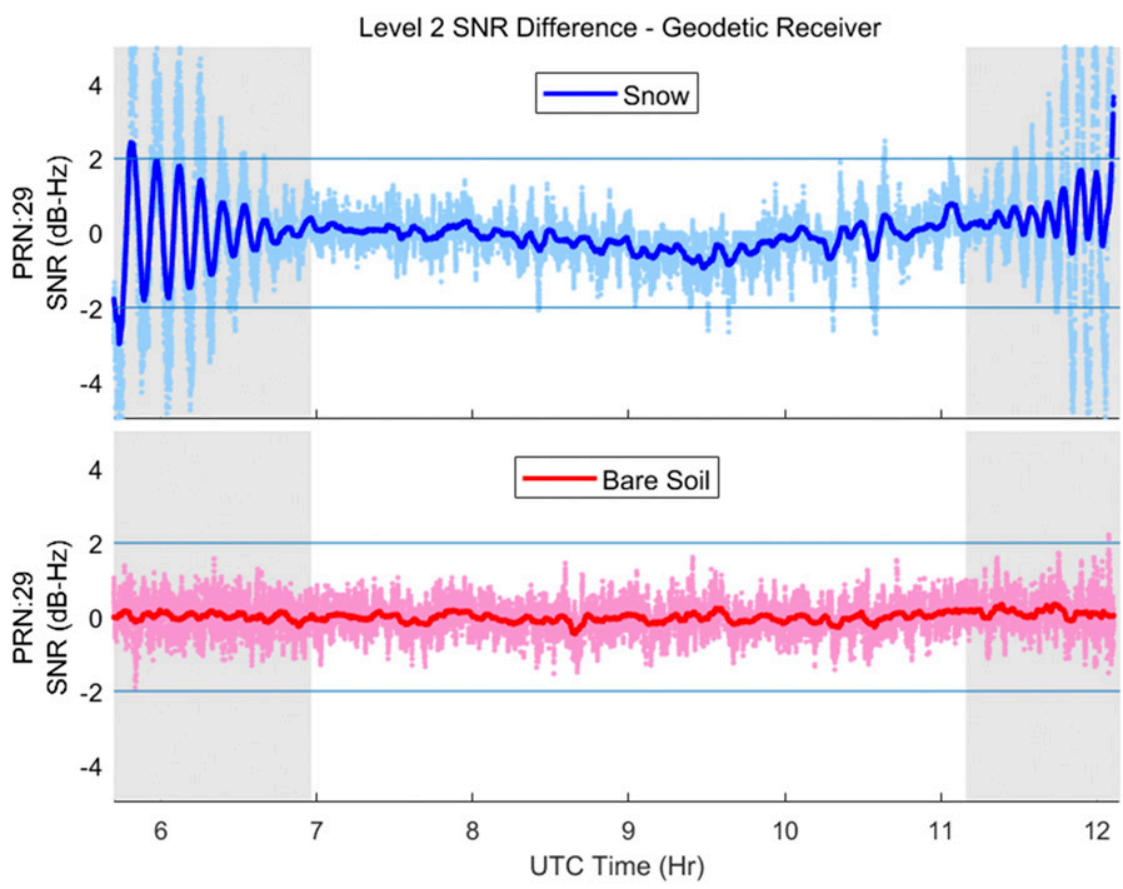

FIG. 11. The level 2 SNR difference on PRN29 from the geodetic grade GPS receiver. (Top) Difference from day 51 (2018) when snow fell at the test site. (bottom) Difference from day 50, when the ground surface was dry. 

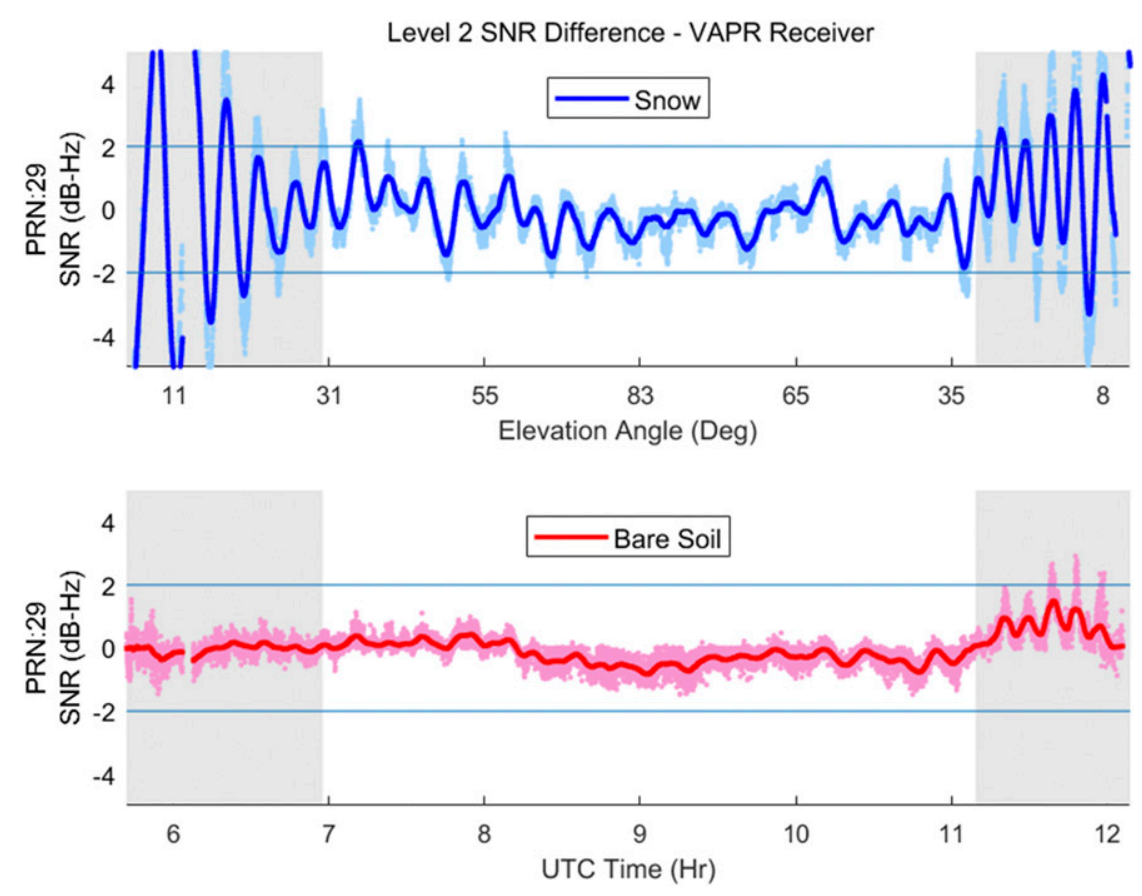

FIG. 12. The level 2 SNR difference on PRN29 from the VAPR receiver. (top) Difference from day 51 (2018) when snow fell at the test site. (bottom) Difference from day 50, when the ground surface was dry. Lighter colors represent the $1-\mathrm{Hz}$ SNR data differenced with the model, darker colors are a 5-min moving average of the difference.

\section{d. Long-term system performance}

The RX11 receiver has been running continuously as part of the VAPR test network since 10 November 2017. Over the 2-month period shown in Fig. 15 the worst-case level 2 difference between the real-time SNR and a prior day model typically stayed well below $2 \mathrm{~dB}-\mathrm{Hz}$. Days with either snowfall or a measurable snow depth were an exception, with a significant number of the level 2 differences reaching the $2 \mathrm{~dB}-\mathrm{Hz}$ detection threshold due both to day to day changes in the ground reflectivity and height as well as to snow on the antenna itself. This again illustrates the challenge of operating an SNR observing GPS receiver without a high-quality multipath suppressing antenna.

Additional outliers unrelated to snowfall occurred on day 346 of 2017 and day 12 of 2018 and show the need for continued testing of the system as part of a larger network of receivers. At these times the threshold on RX11 was exceeded on only one satellite and for several minutes at a time, which is similar to what could be seen during an eruption and would result in a false detection. The drop in observed SNR at these times could have been caused by any temporary obstruction, such as a bird landing on the antenna or nearby foot traffic. The impact of these events is an overall false detection rate of $0.0325 \%$ at the VAPR receiver compared to a rate of $0.0008 \%$ at the geodetic receiver. However, RX06, a VAPR receiver operating on the same network as RX11, was quiet when these events occurred. In an operational network the data from multiple receivers, such as RX06 and RX11, could be combined to decrease the overall rate of false detections due to local obstructions at the receiver sites.

\section{Conclusions}

Early testing has demonstrated the basic operation of the VAPR system and has shown that the performance difference between the navigation grade GPS chips used by VAPR and geodetic grade GPS receivers can be significant due to SNR drift. An additional processing step was added to the ash plume detection technique to remove the bias in SNR observations caused by drift and resulted in improved consistency of the SNR difference. A more fundamental limitation encountered with the SNR data quality was due to the poor multipath signal suppression of low-cost off-the-shelf GPS antennas. Substituting these antennas for larger antennas with high-performance ground planes would offer immediate improvement, though a solution better matched in size and cost with the VAPR node will require further development work. 

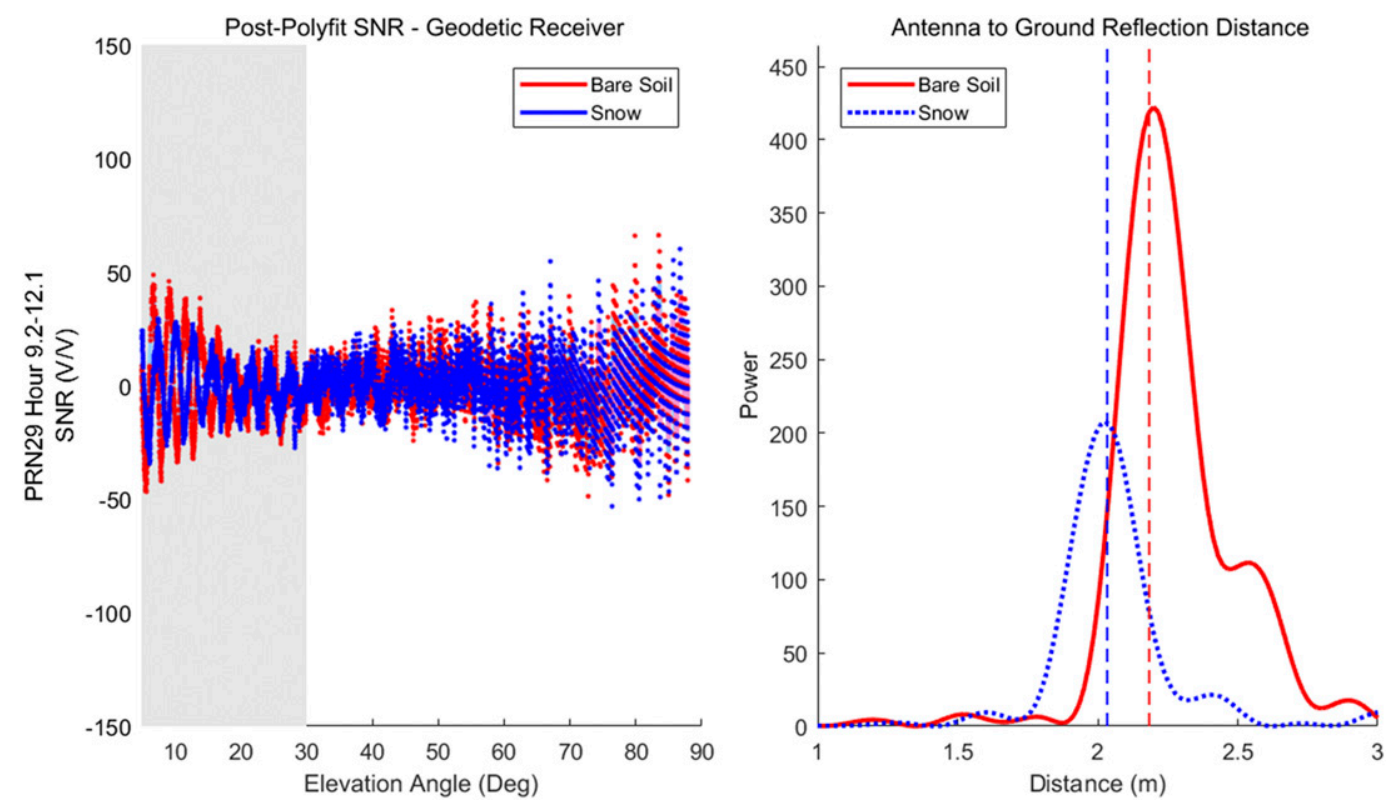

FIG. 13. (left) Geodetic SNR with antenna gain effects removed on day 51 (2018). Gray shading represents a less than $30^{\circ}$ elevation angle. (right) Ground reflection power as a function of height. Dashed vertical lines represent the expected 0.15-m height decrease due to snow depth. Lighter colors represent the 1-Hz SNR data differenced with the model, darker colors are a 5-min moving average of the difference.

In addition, in this paper we evaluated VAPR receiver performance based on the detection strategies used by sparsely placed geodetic quality GPS receivers. However, the VAPR system has been designed to support the collection of GPS SNR data from densely placed VAPR nodes. Further development of a detection algorithm specialized to use data from these densely placed receivers could increase the capability and reliability of the overall system, both by filtering out false detections and by increasing the diversity of
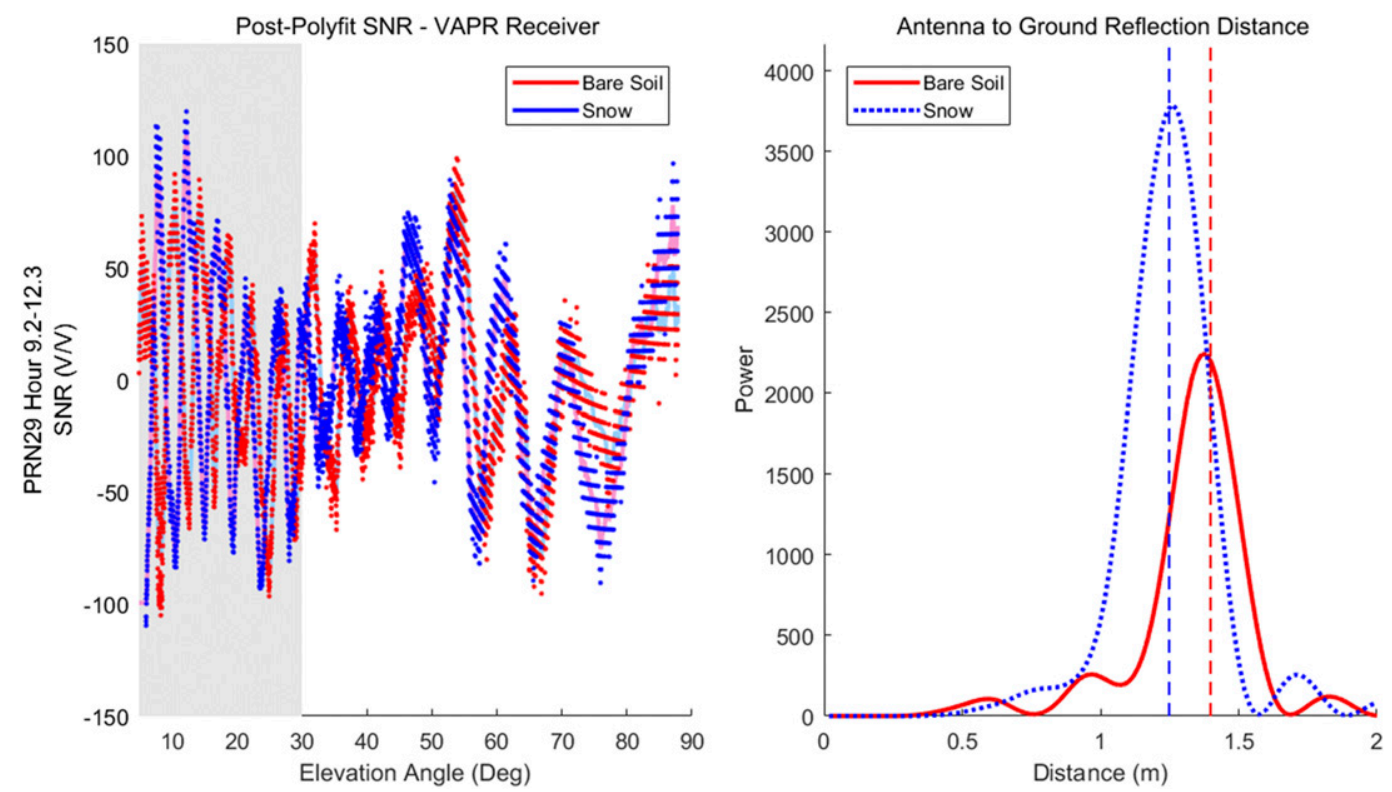

FIG. 14. (left) VAPR SNR with antenna gain effects removed on day 51 (2018). Gray shading represents a less than $30^{\circ}$ elevation angle. (right) Ground reflection power as a function of height. Dashed vertical lines represent the expected 0.15-m height decrease due to snow depth. 


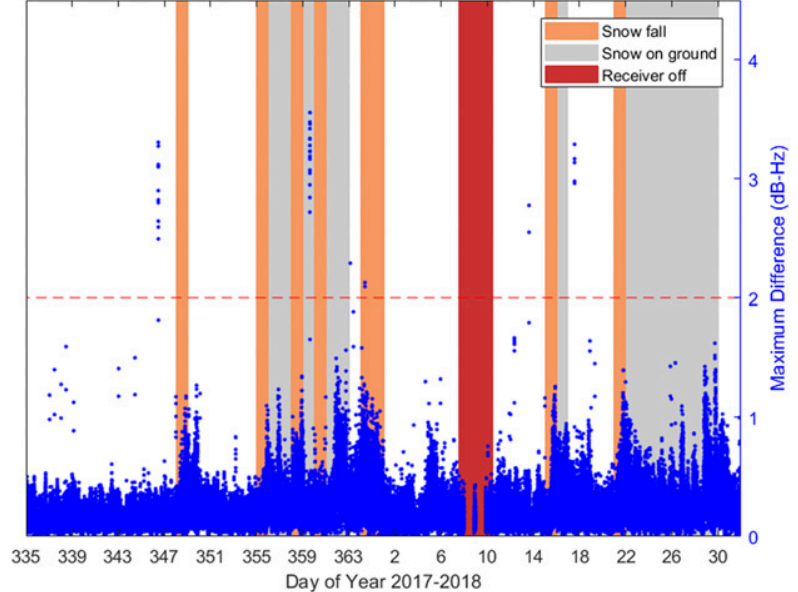

FIG. 15. The maximum difference per minute using the level 2 processing algorithm from the VAPR receiver over the months of December 2017 and January 2018, for all visible satellites. Orange shading represents days with snowfall, gray shading days with snow on the ground, and red shading days when the receiver was temporarily shut down for maintenance.

observation geometries. The dense data would also support ash plume parameter estimation algorithms which could retrieve the eruption time, duration, and plume velocity observations desired by VAACs by timing the intersection of the plume at different heights.

The next step will be to analyze additional data at a system level using SNR observations from multiple node VAPR networks, such as the data collected from a prototype network located at Etna Volcano over the 2017/18 winter season. Work on the detection and estimation algorithms will also continue based on a CU developed GPS/Plume interaction simulation environment. With improved detection and estimation algorithms from this effort and reliable autonomous operation of the VAPR hardware, this system could then provide real-time observations of ash plumes in all weather conditions, for a low cost relative to existing instrumentation.

Acknowledgments. This work has been supported by EAR 1360810 and NASA NNX14AQ14G. We thank Scott Landolt and Justin Lentz at UCAR for supporting field testing at the Marshal Mesa instrumentation site. We thank Nick Matteo for implementing the data storage backend for the real-time VAPR data stream.

\section{REFERENCES}

Agnew, D. C., and K. M. Larson, 2007: Finding the repeat times of the GPS constellation. GPS Solutions, 11, 71-76, https:// doi.org/10.1007/s10291-006-0038-4.
Aranzulla, M., F. Cannavò, and S. Scollo, 2014: Detection of volcanic plumes by GPS: The 23 November 2013 episode on Mt. Etna. Ann. Geophys., 57, 1-6, https://doi.org/10.4401/ ag-6622.

Braun, J., 2011: GPS Soil Moisture Network: MFLEMarshall Field East P.S. GPS/GNSS observations dataset. UNAVCO, accessed 18 July 2018, https://doi.org/10.7283/ T5GT5K9D.

Digi, 2006: Wireless mesh networking: Zigbee vs. DigiMesh. Digi Rep., 6 pp., https://www.digi.com/pdf/wp_zigbeevsdigimesh.pdf.

Donnadieu, F., and Coauthors, 2016: Near-source Doppler radar monitoring of tephra plumes at Etna. J. Volcanol. Geotherm. Res., 312, 26-39, https://doi.org/10.1016/j.jvolgeores. 2016.01.009.

Fournier, N., and A. D. Jolly, 2014: Detecting complex eruption sequence and directionality from high-rate geodetic observations: The August 6, 2012 Te Maari eruption, Tongariro, New Zealand. J. Volcanol. Geotherm. Res., 286, 387-396, https:// doi.org/10.1016/j.jvolgeores.2014.05.021.

Grapenthin, R., J. T. Freymueller, and A. M. Kaufman, 2013: Geodetic observations during the 2009 eruption of Redoubt Volcano, Alaska. J. Volcanol. Geotherm. Res., 259, 115-132, https://doi.org/10.1016/j.jvolgeores.2012.04.021.

Houlié, N., P. Briole, A. Nercessian, and M. Murakami, 2005: Sounding the plume of the 18 August 2000 eruption of Miyakejima volcano (Japan) using GPS. Geophys. Res. Lett., 32, L05302, https://doi.org/ 10.1029/2004GL021728.

Joseph, A., 2010: Measuring GNSS signal strength. InsideGNSS, Nov/Dec, 20-25, https://insidegnss.com/measuring-gnsssignal-strength/.

Larson, K. M., 2013: A new way to detect volcanic plumes. Geophys. Res. Lett., 40, 2657-2660, https://doi.org/10.1002/ grl.50556.

__ and F. G. Nievinski, 2013: GPS snow sensing: Results from the EarthScope Plate Boundary Observatory. GPS Solutions, 17, 41-52, https://doi.org/10.1007/s10291-0120259-7.

, S. Palo, C. Roesler, M. Mattia, V. Bruno, M. Coltelli, and D. Fee, 2017: Detection of plumes at Redoubt and Etna volcanoes using the GPS SNR method. J. Volcanol. Geotherm. Res., 344, 26-39, https://doi.org/10.1016/j.jvolgeores.2017.04.005.

Mackie, S., K. Cashman, H. Ricketts, A. Rust, and M. Watson, Eds., 2016: Volcanic Ash: Hazard Observation. Elsevier, 300 pp., https://doi.org/10.1016/C2014-0-03381-3.

Mastin, L. G., A. R. V. Eaton, D. J. Schneider, and R. P. Denlinger, 2017: Ongoing efforts to make ash-cloud model forecasts more accurate. NATO STO Doc. STO-MP-AVT-272, 12 pp.

Misra, P., and P. Enge, 2006: Global Positioning System: Signal, Measurements, and Performance. 2nd ed. Ganga-Jumana Press, 569 pp.

Montopoli, M., 2016: Velocity profiles inside volcanic clouds from three-dimensional scanning microwave dual-polarization Doppler radars. J. Geophys. Res. Atmos., 121, 7881-7900, https://doi.org/ 10.1002/2015JD023464.

National Oceanic and Atmospheric Administration, 2018: Boulder daily weather data from the NOAA/NWS cooperative site. Accessed 6 February 2019, https://www.esrl.noaa.gov/psd/ boulder/data.daily.html.

Nievinski, F. G., and K. M. Larson, 2014a: Inverse modeling of GPS multipath for snow depth estimation-Part I: Formulation and simulations. IEEE Trans. Geosci. Remote Sens., 52, 6555-6563, https://doi.org/10.1109/TGRS. 2013.2297681. 
_, and _ 2014b: Inverse modeling of GPS multipath for snow depth estimation-Part II: Application and validation. IEEE Trans. Geosci. Remote Sens., 52, 6564-6573, https://doi.org/ 10.1109/TGRS.2013.2297688.

Ohta, Y., and M. Iguchi, 2015: Advective diffusion of volcanic plume captured by dense GNSS network around Sakurajima volcano: A case study of the vulcanian eruption on July 24, 2012. Earth Planets Space, 67, 157, https://doi.org/10.1186/ s40623-015-0324-x.
Petersen, G. N., 2010: A short meteorological overview of the Eyjafjallajökull eruption 14 April-23 May 2010. Weather, 65, 203-207, https://doi.org/10.1002/wea.634.

Witham, C., M. Hort, D. Thomson, S. Leadbetter, B. Devenish, H. Webster, F. Beckett, and N. Kristiansen, 2017: The current volcanic ash modelling set-up at the London VAAC. Met Office Tech. Rep., 9 pp., https:/www.metoffice.gov.uk/binaries/content/ assets/mohippo/pdf/aviation/vaac/london_vaac_current_modelling setup_nov17.pdf. 